Proceedings of the 35th European Peptide Symposium

Patrick B. Timmons, Chandralal M. Hewage, Michal Lebl (Editors)

European Peptide Society \& PSP, 2018

\title{
Imaging evaluation of an in vivo long-acting Neuropeptide(NPY) analogue for multimodality breast tumor therapy
}

Ming-Hsin Li, Su-Jung Chen, Ming-Wei Chen, Chun-Fang Feng, Yuan-Ruei Huang, Sheng-Nan Lo, Cheng-Hui Chuang, Shih-Ying Lee, Chih-Hsien Chang

Institute of Nuclear Energy Research, Taiwan

https://doi.org/10.17952/35EPS.2018.295

Neuropeptide Y (NPY) is a 36-amino acid peptide and regulates in various physiological functions through its four receptors. Recently, NPY receptor- Y1R has been found to be overexpressed in breast carcinomas. Although the truncated NPY analog has high affinity with Y1R, it is rapidly metabolized, resulting in low tumor uptake. The aims of this study were to synthesize a long-circulated NPY analog and evaluate tumor image of the $4 \mathrm{~T} 1$ animal model as a candidate for breast cancer therapy.

\section{Materials and Methods}

We synthesized INER-7218, which is a 16-amino acid NPY analog. Its structure consists of three parts-(1) chelate for radiolabeling(2) Long-acting linker (3)NPY analogue for high affinity with breast cancer. In the radiolabeling procedure, amount of INER-7218 was dissolved in buffer, followed by addition of (3-12mCi) In-111 or Lu-177, and incubated in high temperature. The labeling efficiency was determined by Radio-TLC. The Radio-HPLC are used for analyzing labeling purity. In animal study, tumor xenografts were performed in 6-wk-old female BALB/c mice by subcutaneous injection of $2^{*} 10^{6} 4 \mathrm{~T} 1$ cells, and nanoSPECT/CT imaging was performed at $0.5 \mathrm{~h}$ to $48 \mathrm{~h}$ after injecting of the ${ }^{111} \mathrm{In}$ - INER-7218

\section{Results}

We can get the high labeling efficiency ( $>90 \%)$ of Lu-177- INER-7218, and In-177- INER-7218 after reacting 15-30min. In vivo study, the nanoSPECT/CT image of the 4T1 animal model revealed that In-111-INER-7218 has with high tumor uptake value (ID\%/g $>10)$ and high tumor to muscle $(\mathrm{T} / \mathrm{M}>9)$ ratio in 48 hours after tail vein injection

\section{Conclusion}

The result shows the INER-7218 is easy to radiolabel with Lu-177 and In-111. The two compounds are high labeling efficiency and high resolution of the image. From the in vivo study, we consider the INER-7218 as a long circulation radiopharmaceutical candidate for companion diagnostics (CDx). Furthermore, we apply such INER-7218 for the design of multimodality carriers. Peptide Receptor Radionuclide Therapy (PRRT) is a widely known molecular targeted therapy. PRRT is performed by using a small peptide which is combined with radionuclides.

INER-7218 is not only a good tracer but also a therapeutic drug by labeling with Lu177.

The capabilities of INER-7218 make it a potential drug for multimodality breast tumor therapy.
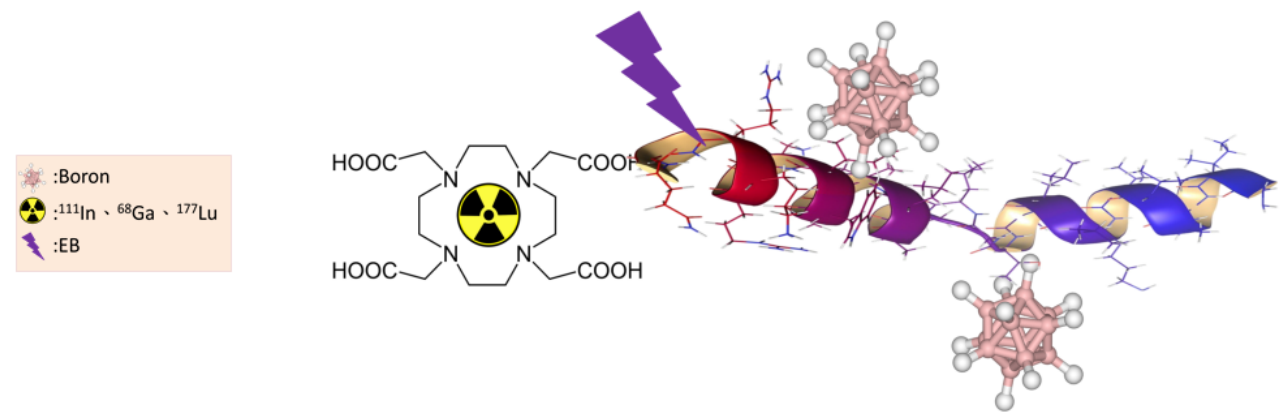

Figure 1: Schematic diagram of Radiolabelled INER-7218 labelled 
(A)

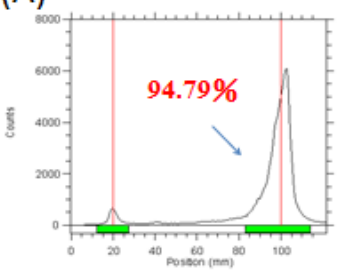

(B)

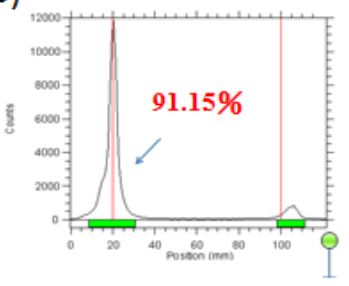

(C)

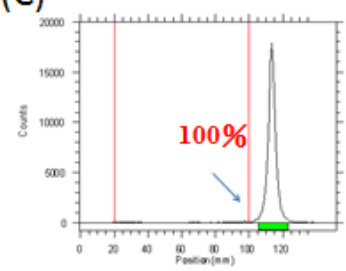

(D)

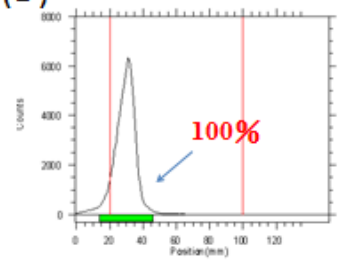

(E)

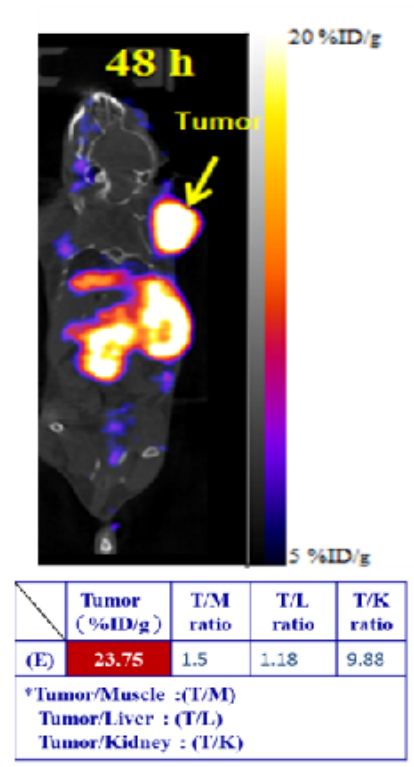

Figure 2: The labeling efficiency is analyzed by Radio-TLC. (A) Free In-111 at solvent front.(B) 111In-INER$7218(R f=0)$, the labeling efficacy is $91.15 \%$. (C) Free Lu-177 at solvent front.(D) 177Lu-INER-7218 (Rf=0), the labeling efficacy is $100 \%$. 